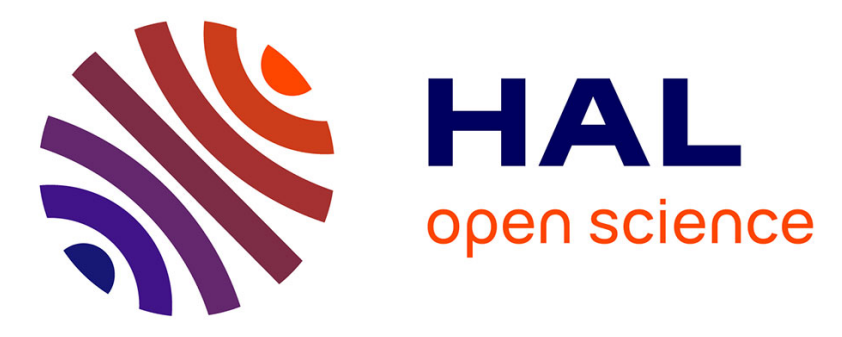

\title{
Hydraulically-actuated compliant revolute joint for medical robotic systems based on multimaterial additive manufacturing
}

Antoine Pfeil, Marius Siegfarth, Francois Geiskopf, Tim Philipp Pusch, Laurent Barbé, Pierre Renaud

\section{To cite this version:}

Antoine Pfeil, Marius Siegfarth, Francois Geiskopf, Tim Philipp Pusch, Laurent Barbé, et al.. Hydraulically-actuated compliant revolute joint for medical robotic systems based on multimaterial additive manufacturing. IEEE International Conference on Robotics and Automation (ICRA), Jan 2019, Montréal, Canada. hal-02369392

\section{HAL Id: hal-02369392 \\ https://hal.science/hal-02369392}

Submitted on 18 Nov 2019

HAL is a multi-disciplinary open access archive for the deposit and dissemination of scientific research documents, whether they are published or not. The documents may come from teaching and research institutions in France or abroad, or from public or private research centers.
L'archive ouverte pluridisciplinaire HAL, est destinée au dépôt et à la diffusion de documents scientifiques de niveau recherche, publiés ou non, émanant des établissements d'enseignement et de recherche français ou étrangers, des laboratoires publics ou privés. 


\title{
Hydraulically-actuated compliant revolute joint for medical robotic systems based on multimaterial additive manufacturing (Author manuscript, publisher version DOI: 10.1109/ICRA.2019.8793666)
}

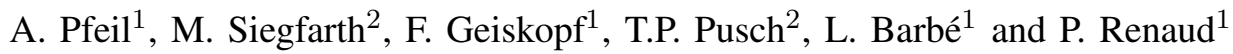

\begin{abstract}
In this paper, an active compliant revolute joint actuated by hydraulic energy is developed. The joint is made of polymer for integration in medical robotic systems, even in a challenging environment such as Magnetic Resonance Imaging (MRI). The use of multimaterial additive manufacturing allows us to develop two original aspects. First, a new seal design is proposed to build miniature hydraulic cylinders embedded in the active joint, with low level of friction. Second, a rackand-pinion mechanism is being integrated to a compliant revolute joint to obtain a high level of compactness. Design and experimental assessment of the hydraulic cylinder and the compliant joint with embedded rack-and-pinion are presented, as well as an illustration in the context of needle manipulation with passive teleoperation.
\end{abstract}

\section{INTRODUCTION}

Image-guided procedures as performed in interventional radiology are of great interest for their minimal invasiveness and the achievable accuracy. Patient access when using CT or MR scanners is however difficult [1]. Robotic assistance constitutes a solution that can in addition solve the problem of staff exposure to X-rays of CT or cone beam CT scanners [2]. Robotic devices must not alter medical image quality and need to be compact enough to fit in the scanner bore together with the patient. This is particularly challenging for MRguided procedures [3] and in the following we consider the design of actuation solutions for robotic systems in this context. Numerous robotic architectures are based on active rotary motions. We therefore focus our analysis on the implementation of active revolute joints.

Several works have shown the advantages of fluidic actuation using pneumatic or hydraulic energy. Hydraulic solutions are of particular interest as fluid incompressibility makes control easier and high power density can be obtained [4]. Stepper motors were proposed [5], [6], for which speed and resolution can be difficult to satisfy simultaneously. Balloonbased design introduced in [7], that can be associated to flexible fluidic actuators, offers a continuous motion. Such

This work was supported by the INTERREG Upper Rhine program from the ERDF (European Regional Development Fund), SPIRITS project, as well as the Investissements d'Avenir (Robotex ANR-10-EQPX-44, Labex CAMI ANR-11- LABX-0004).

${ }^{1}$ A. Pfeil, F. Geiskopf, L. Barbé and P. Renaud are with ICube, University of Strasbourg - INSA Strasbourg, France a.pfeileunistra.fr

${ }^{2}$ M. Siegfarth, T.P. Pusch are with the Fraunhofer Institute for Manufacturing Engineering and Automation, Project Group for Automation in Medicine and Biotechnology, Mannheim, Germany marius.siegfarth@ipa.fraunhofer.de

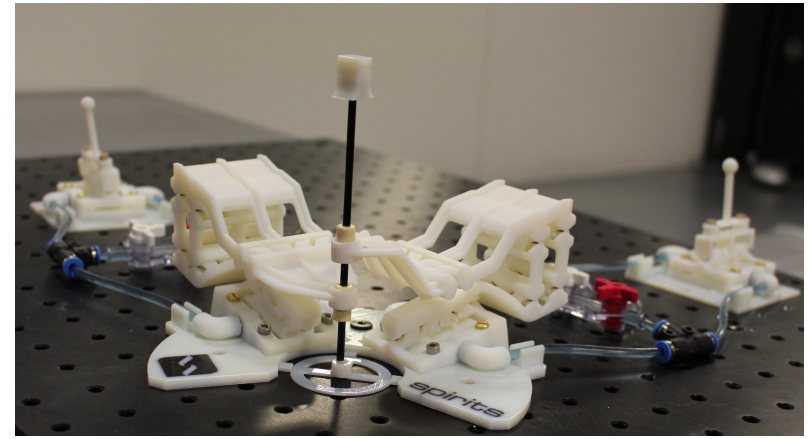

Fig. 1: Proof of concept built using the proposed hydraulically-actuated compliant revolute joint: a needle manipulation system with passive teleoperation.

design is however delicate to implement to avoid control issues in presence of nonlinearities [8]. In several works [9], [10], [11], fluidic energy is more simply used to produce a translation, using a cylinder, that is converted into a rotation using a transformation mechanism. The main issue for cylinder design is the sealing. With pneumatic energy, sealless solution was tested [6] that seems difficult to adopt for hydraulic cylinders. Friction of rubber seals usually adopted in cylinders can be lowered using rolling diaphragms [4], [12]. The issue is then the miniaturization of such components with large stroke to diameter ratios. In [13] it was shown that additive manufacturing can be adopted to produce soft materials compatible with the sealing function. For medical robotic systems, additive manufacturing of polymer structures is of interest for image compatibility, compactness and also weight in case of patient-mounted devices [14], [2]. In this paper, we therefore investigate its use to produce seals for miniature hydraulic cylinders. Our first contribution is to design seals integrated to the pistons thanks to multimaterial additive manufacturing (MMAM), which allows for adjusting friction characteristics through geometries that cannot be implemented using conventional seals.

Transmission belts, rack-and-pinion systems are well-known solutions to convert cylinder translation into a rotation [4], [11]. They can offer high mechanical efficiency, but compactness can be an issue using standard components, as observed in [15] with hydraulic actuation unit separate from robotic device. In [16], additive manufacturing (AM) allowed the 
design of very compact transformation mechanism thanks to the freedom of shape in part design. We previously developed a compliant revolute joint, the HSC joint [17], using MMAM. Range of motion and lifetime are adapted to medical robots of limited number of uses. It was in addition recently shown that the materials could also endure gamma sterilization [18]. Our second contribution is thus the design of a transformation mechanism in the HSC joint to finally get a hydraulically-actuated revolute joint. The freedom of shape offered by MMAM is exploited to maximize compactness. As a third contribution, we illustrate the possible use with a 2 degree-of-freedom needle manipulator providing a remote center of motion, shown Figure 1.

Design and testing of the HSC joint with integrated transformation mechanism is described in section II. Development of sealing solutions using MMAM for miniature hydraulic cylinders is then introduced in section III. Integration of elements to build an active revolute joint is presented in section IV as well as an illustration in the context of needle manipulation, before giving conclusions and perspectives in section V.

\section{DESIGN OF MMAM-BASED REVOLUTE JOINT WITH EMBEDDED TRANSFORMATION MECHANISM}

\section{A. MMAM Process}

Joint design is closely linked to the manufacturing process specifications in terms of material properties and geometrical accuracy. The MMAM process we consider is Polyjet (Stratasys Ltd, USA), based on photopolymerization. It allows the production of parts composed of 2 materials. As described in [17], we consider the use of VeroWhite and TangoBlack Plus materials, following commercial denominations. The first material can be considered as a rigid polymer and the second one as an elastomer. Given its quasi-incompressible behavior in compression, the TangoBlack Plus material will also be designated as a rubber-like material.

Using a standard Connex 350 system, layer thickness during part production is equal to 30 microns, and in-plane resolution to 42 microns. Internal experiments show the manufacturing accuracy is between 10 and 100 microns in standard conditions, with a minimal size of geometrical features equal to $1 \mathrm{~mm}$.

\section{B. Design principle}

MMAM process offers freedom of shape in part design and the possibility to combine rigid and flexible materials. Both features are exploited in the design of the HSC revolute joint [17]. The design approach consisted then of creating a 3D shape from a 2D section and a helical sweep (Figure 2), usually not adapted to manufacturing with conventional techniques. The rubber-like material in the center allows for the rotation of volume 1 , while the helical sweep avoids motions other than rotation, due to the incompressible behavior of TangoBlack Plus [17]. The range of motion is defined by the profile with the angle $\alpha$ (Figure 4a). Two helices of opposite pitches (Figure 2) are used to cancel the translation that could occur due to the helicoidal shape. Detailed analysis of the

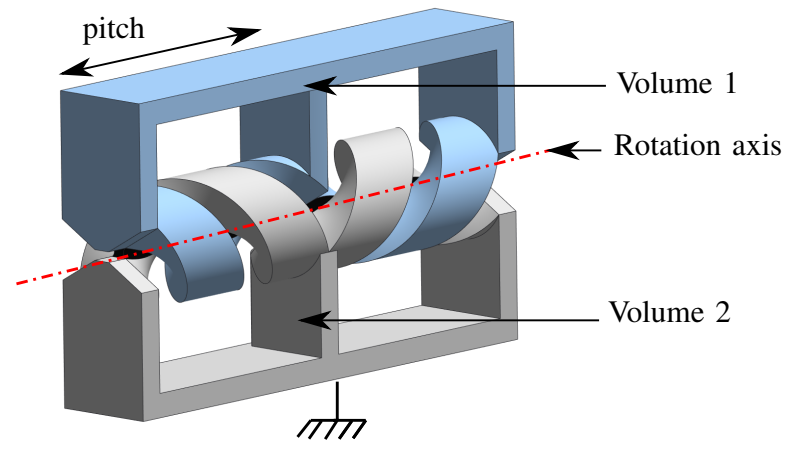

Fig. 2: Original HSC design.

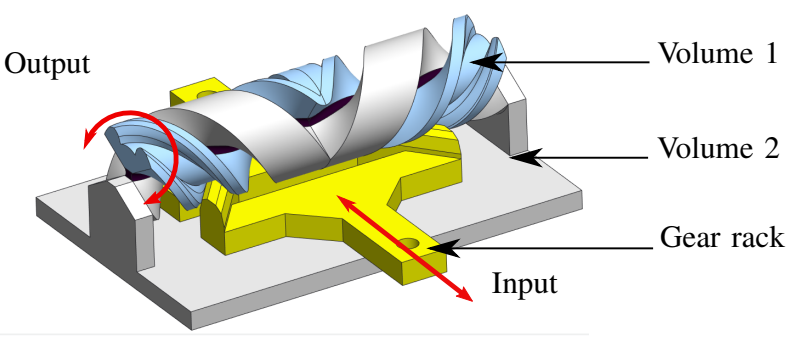

Fig. 3: HSC joint with embedded rack-and-pinion system.

joint behavior [17] showed the rubber-like material thickness $e$ has to be minimized and that increasing the pitch value helps improving the joint kinematic behavior.

The shape of the volumes 1 and 2 (Figure 2) does not impact the joint rotation as long as their lateral surfaces are not modified. To actuate the joint, our proposition is therefore to modify the moving volume (volume 1) to integrate a rack-and-pinion system (Figure 3). The gear rack is the input element. It is in translation with respect to the volume 2. The output element is the pinion, embedded in volume 1. The solution has 3 main advantages: i) the pinion is integrated in the joint, which is in favor of compactness. ii) the transmission mechanism is not friction-based, which is in favor of mechanical efficiency, iii) as the joint is composed of two helical shapes with opposite pitches, gear integration leads to a helical rack and pinion that can be advantageous in terms of transmission accuracy. The presence of two symmetrical helical shapes allows us to avoid at the same time axial forces on the joint that would be generated by a

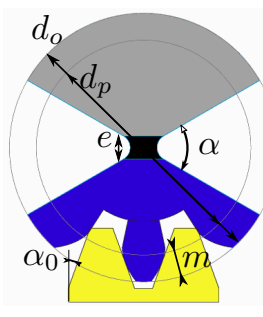

(a)

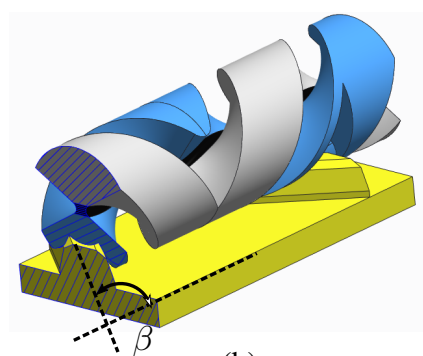

(b)
Fig. 4: (a) Gear and joint design parameters, (b) 3D view of the rack-and-pinion system with section of the gear. 
single helical gear system.

\section{Gear design}

Gear design is performed considering the manufacturing process performances and the search for compact solutions. The main design parameters (Figure 4) of a normalized helical involute gear profile are the pressure angle $\alpha_{0}$, the module $m$, the helix angle $\beta$ and the number $Z$ of teeth of the gear. Here, a standard pressure angle of $20^{\circ}$ is adopted. The helix angle $\beta$ is correlated to the pitch value of the helices in the HSC joint: $\tan (\beta)=\pi * \mathrm{~d}_{\mathrm{p}} /$ pitch with $d_{p}=m . Z$ the pitch diameter of the gear. The pitch value of the joint helices and their outer diameter have a direct impact on the compactness and should be minimized. They also affect the volume available to integrate the gear. Given manufacturing specifications, the pitch value is set to $20 \mathrm{~mm}$, the outer diameter $d_{o}$ to $10 \mathrm{~mm}$, and the pitch diameter of the gear is determined from the analysis of tooth geometry.

Tooth geometry is controlled by the number of teeth $Z$ and the module $m$, with the possibility to include addendum modification [19]. We tested all possible values of $Z$, but we did not find any standard solution for the selected outer diameter: the size of the tooth at its bottom is then not sufficient for production. Addendum modification would lead to top land thickness below $1 \mathrm{~mm}$, which is again not acceptable. The solution we adopt is to use a contact between one tooth and the gear rack, the tooth being however divided in two, to have two half teeth in contact with the gear rack (Figure $4 \mathrm{~b}$ ). With $m=1, Z=8, \beta=51.5^{\circ}$, we have a range of motion of $\pm 45^{\circ}$, with an overlap ratio of 1.25 .

The angle $\alpha$ of the compliant joint is chosen equal to $60^{\circ}$. The CAD design is presented on Figure 3. The presence of two contact areas between the gear rack and the HSC joint allow us to guide the gear rack by means of a planar joint with respect to the base. The gear rack and the compliant joint can then be printed as two separate elements, with the rack inserted afterwards.

\section{Experimental assessment}

An experimental evaluation is performed to validate the solution, in particular concerning the input-output relationship in terms of displacement and force, and also presence of backlash with production using MMAM. Quasi-static testing is adopted, which is not restrictive given the intended applications.

To assess the input-output relationship, load on the gear rack is applied by means of weights connected to the rack using a cable-pulley system (Figure 5). Displacement of the gear rack is measured with a laser-based sensor (optoNCDT 1420, MicroEpsilon). A pulley is added to the gear embedded in the HSC joint to generate a torque. A linear table (FB075, Nanomotion) equipped with a force sensor (K1107, SCAIME) is then used to pull on a cable fixed to the pulley. Position of input and output elements are then known, as well as force and torque applied on them. As a cable is being used, a tensioning phase has to be performed, where no gear rack displacement is noticed.

\begin{tabular}{l|c|c} 
Load & Measured force & Theoretical value \\
\hline 3.3 & $\mathbf{2 . 4 9}(0.24)$ & 1.8 \\
6.6 & $\mathbf{4 . 4 9}(0.25)$ & 3.6 \\
9.9 & $\mathbf{6 . 7 4}(0.36)$ & 5.3
\end{tabular}

TABLE I: Evaluation of force transmission. All values in Newtons. Mean (standard deviation) values are reported for experimental results.

In the following, this phase is not represented as it is not linked to the joint behavior.

The pulley added to the gear has a diameter of $D_{p u l}$ $=14.7 \mathrm{~mm}$. Given the gear primitive diameter is equal to $D_{p}=8 \mathrm{~mm}$, the displacements of the cables placed at the input and output sides are linked by a ratio $k=D_{p} / D_{p u l}=$ 0.54. Figure 6 shows the gear rack displacement versus the displacement of the linear table for three loads applied on the gear rack. Slight non-linearity is observed initially, that may be due to the joint compliance or the setup flexibility. The input-output relationship is linear, with a slope of 0.59 for all the plotted curves, which is close to the theoretical value of 0.54 . The range of displacement is a bit lowered when the load is increased. This might be due to early contacts between the helicoidal surfaces of the HSC joint, as the compliant joint is not submitted to a pure torque, and some parasitic displacement probably appears. However, the overall rack displacement range is still above $5.5 \mathrm{~mm}$, which correspond to angular range of motion of $\pm 39^{\circ}$, in adequacy with our need for applicative contexts. Mean force and standard deviation for the different loads applied on the rack are given in Table I. The standard deviations are in the range of $5 \%$ to $10 \%$ of the mean value, which shows that the force variations over the joint range of motion are small. Theoretical values of forces can be computed with the assumption of a perfect transmission by multiplying the force applied to the rack by the transmission ratio $k$. Differences between measures and theoretical values are between $0.8 \mathrm{~N}$ and $1.8 \mathrm{~N}$, in the absence of any lubrication of specific preparation of $3 \mathrm{D}$ printed parts.

For backlash evaluation, a specific version of the compliant joint is produced with the pinion connected to the base. A linear motion is applied on the gear rack by means of a linear table equipped with a force sensor. Backlash is detected as a non-linearity in the force measurement, since the rack moves freely before the force increases. The recorded force-displacement profile once we take up play between gear and rack allows us in addition to determine the component stiffness. Backlash is evaluated equal to \pm 0.05 $\mathrm{mm}$ that corresponds to backlash output of $\pm 0.75^{\circ}$ and stiffness to $30 \mathrm{~N} . \mathrm{mm}^{-1}$ at the rack level, which corresponds to $480 \mathrm{~N} \cdot \mathrm{mm} \cdot \mathrm{rad}^{-1}$ at the pinion level. As the designed gearrack mechanism displays interesting performances, hydraulic cylinders to provide the translational motion to the rack are developed. 


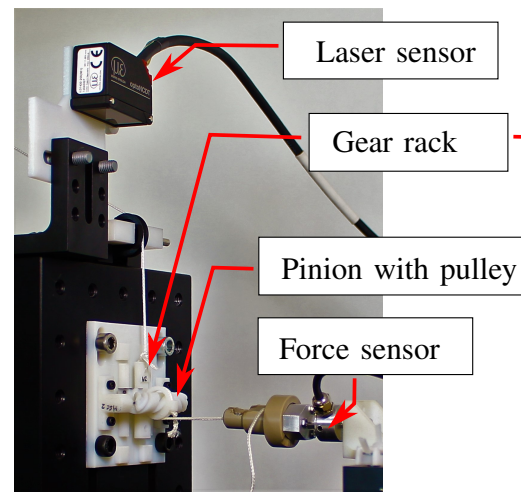

(a)

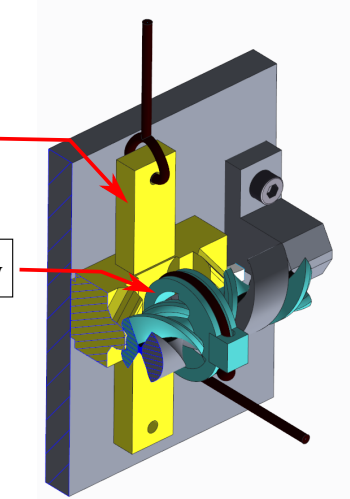

(b)
Fig. 5: Experimental setup for input-output displacement/force evaluation. (a) Photograph (b) CAD view of pulley integrated to the gear

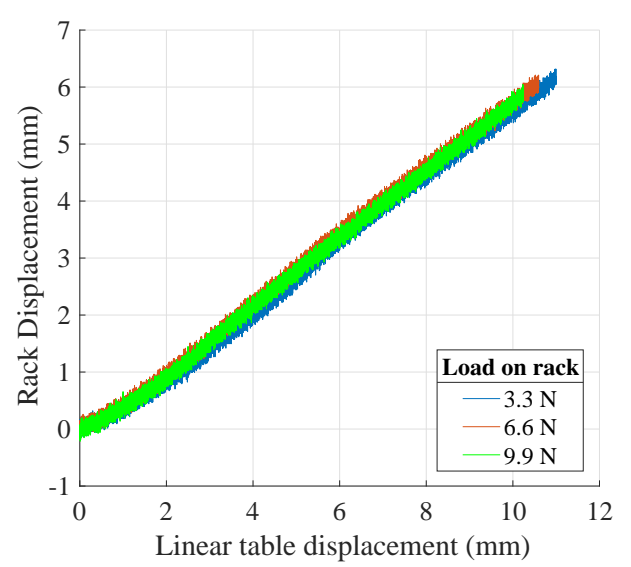

Fig. 6: Input-output displacement relationship.

\section{HYDRAULIC TRANSMISSION DESIGN}

\section{A. Piston Design}

A single-acting piston is considered, its integration to the joint being discussed in section IV. The piston efficiency is linked to the sealing performances. Absence of leakage under pressure is needed, and low friction forces are desired to ease the control and to improve the mechanical efficiency. Making use of the MMAM capabilities, the piston sealing is designed to be embedded in the piston. Three integrated sealing designs have been conceptualized. One corresponds to a conventional O-ring seal but embedded in the piston ("Full" design in Figures 7 and 8). The other two designs introduce in addition deformable shapes of seal so the fluid pressure can exert radial forces on the seal in order to have variable radial forces to maintain the seal efficiency. One design is the same as the "Full" design, but with a hollow head ("Hollow" design), the other one is based on an X-ring design ("X-ring"). For reference, a 2-part design ("O-ring") is also considered with a standard commercial O-ring made out of nitrile butadiene rubber, with Shore Hardness 70, mounted on a 3D-printed piston produced using SLA (Formlabs Form 2, FLGPWH03). The groove depth

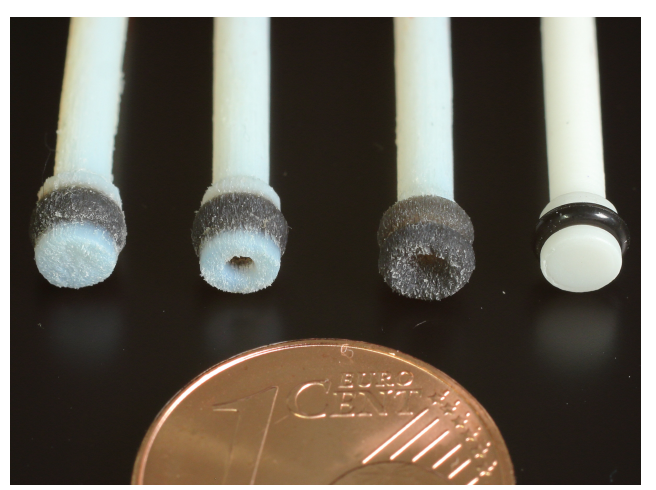

Fig. 7: Piston designs "Full", "Hollow", "X-ring" and "Oring".

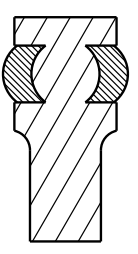

(a)

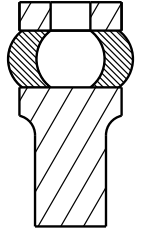

(b)

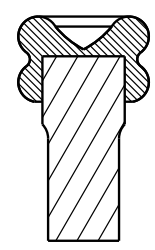

(c)

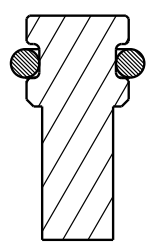

(d)
Fig. 8: CAD detail of the piston sealings (a) "Full", (b) "Hollow", (c) "X-ring" and (d) "O-ring".

of the piston is designed such that the outer diameter of the mounted O-ring equals that of the printed seals, thus deviating from a standard O-ring groove design. In order to compare the integrated sealings with the commercial O-ring, the integrated sealings are produced with a digital material (DMShore70) obtained by combination of TangoBlack Plus and VeroWhite materials to get the same hardness.

For all designs, the pistons are intended to be used with a cylinder barrel of $4 \mathrm{~mm}$ inner diameter. The piston rod diameter is equal to $2.5 \mathrm{~mm}$. Such small dimensions are of interest for the compactness of the active joint and allows consistent production of pistons with Polyjet process, as verified by optical measurements of piston rods that showed standard deviation in the order of $0.05 \mathrm{~mm}$. The cylinder barrel is a polymer sliding bearing with $4 \mathrm{~mm}$ inner diameter (Igus H370SM-0405-12), preferred to 3D printed barrel for the better surface roughness.

It is difficult to use modeling to predict the best piston diameter for sealing. As a consequence, an experimental evaluation was conducted. Each design is assessed in terms of fluid leakage and friction using three sealing sizes. Outer sealing diameters are chosen equal to $4.4,4.5$ and $4.6 \mathrm{~mm}$ for the integrated geometries. For the conventional 2-part design, an O-ring of $2.2 \mathrm{~mm}$ inner diameter and 1-mm thickness is mounted on pistons with inner slot diameters equal to 2.6, 2.7 and $2.85 \mathrm{~mm}$. These sizes were selected in a preliminary study for the absence of noticeable leakage during visual inspection. 

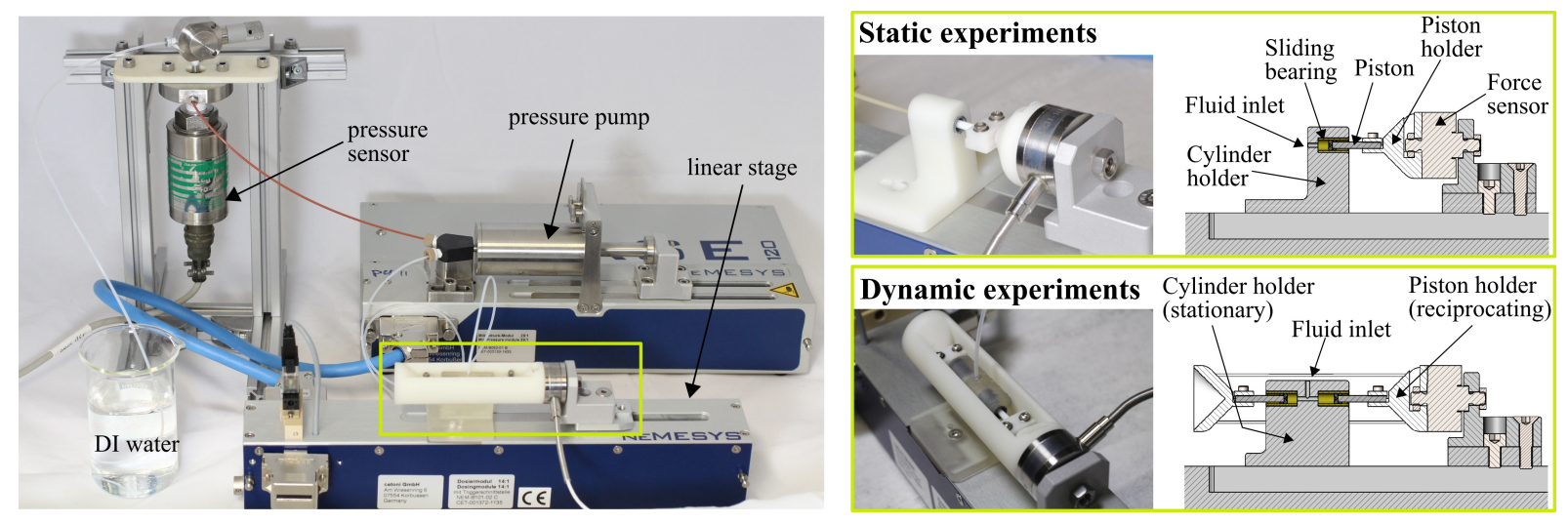

Fig. 9: General view of set-ups used for static and dynamic measurements.

\section{B. Experimental Setup and Protocol}

The three sizes of each piston introduced above are designated in the following as "S", "M" or "L" size. For each size of each design, four samples are produced. Two experimental setups are being used for evaluation of static and dynamic behavior.

1) Static assessment: In the first setup (Figure 9), a piston is placed in a piston holder and inserted into the cylinder. The piston holder is mounted on a precision syringe pump (NeMESYS, Cetoni GmbH, Germany) through a force sensor (Burster 8431). Deionized water (DI) water is sent into the barrel by means of a second precision syringe pump of same reference. $5 \mathrm{ml}$ of air were added to the DI water in order to allow for smooth manual control of the pressure applied by the pump.

The pressure acting on the piston is increased incrementally from $0 \mathrm{MPa}$ to $1.5 \mathrm{MPa}$ while measuring the resulting force as well as monitoring the pressure in the system. After having reached the desired pressure increment, the pressure is kept constant for ten seconds, followed by a one-minute pause after the last increment. If leakage leads to a pressure drop over $0.1 \mathrm{MPa}$, the experimental run is aborted. Each test allows us to evaluate the presence of leakage and the ratio between pressure and generated forces.

2) Dynamic assessment: The same instrumentation is being used for dynamic evaluation. Two cylinders are tested at the same time, the pistons being connected altogether by means of an additional part as represented in Figure 9. Because of the setup symmetry, the application of pressurized fluid does not generate forces on the force sensor. The setup allows us to have controlled displacements of the pistons using one precision syringe pump, and to apply different fluid pressures controlled using the second pump. Thus, the forces related to the friction between the pistons and the cylinders can be evaluated.

The motion profile consists of three velocities $(1,2 \& 3$ $\mathrm{mm} / \mathrm{s}$ ) in the two directions. Its duration corresponds to a travel distance of $6 \mathrm{~mm}$ for each velocity along with a $2 \mathrm{~s}$ pause between each of the velocities. The profile is repeated three times for one measurement cycle, adding up to a total duration of $100 \mathrm{~s}$. If the sealings show no significant leakage,

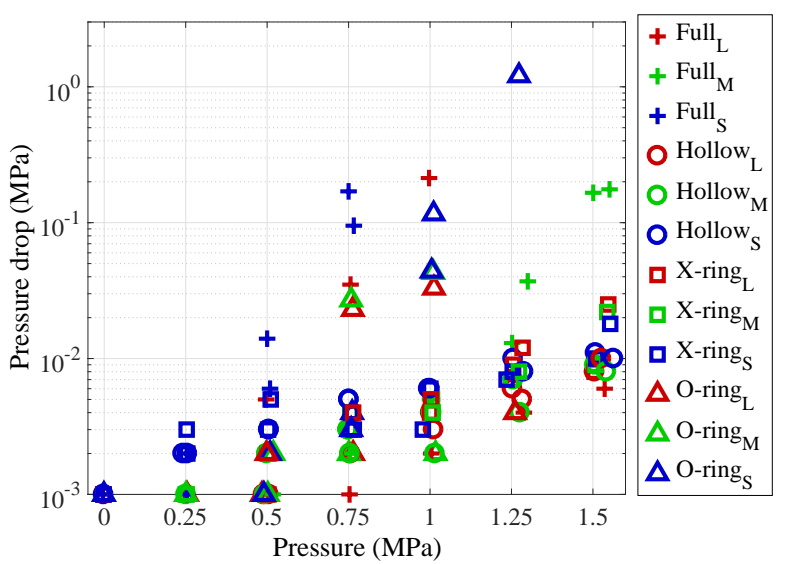

Fig. 10: Pressure drop during dynamic experiments.

i.e. the pressure does not drop more than $0.1 \mathrm{MPa}$, the pressure is increased to the next increment in steps of 0.25 $\mathrm{MPa}$ up to 1.5 MPa. The experimental run is continued in this fashion until either leakage occurs or all pressure increments are completed successfully.

\section{Results and Discussion}

1) Leakage: During static evaluation, the Full ${ }_{S}$ pistons were the only ones to present significant pressure drops. Pressure drop values for one experiment cycle during the dynamic assessment are represented in Figure 10. As expected, pressure drop increases if seal size is lowered. It is interesting to note that the drop level is generally below $0.01 \mathrm{MPa}$, except for Full and O-ring designs. In the O-ring experiments, leakage indeed occurs when the pressure is high enough to partially squeeze the O-ring into the gap between the piston and the cylinder. Alternate designs, with hollow O-ring and X-ring however offer interesting performances: with a pressure of $1.5 \mathrm{Mpa}$, the highest pressure drop is $0.025 \mathrm{MPa}$, which represents $1.7 \%$ of the pressure.

2) Force generation: From static assessment, the ratio between pressure and generated force is calculated for each piston by dividing the measured force by the pressure at the point of highest pressure during one test. Mean values for the four pistons per geometry and size are given in table II. 


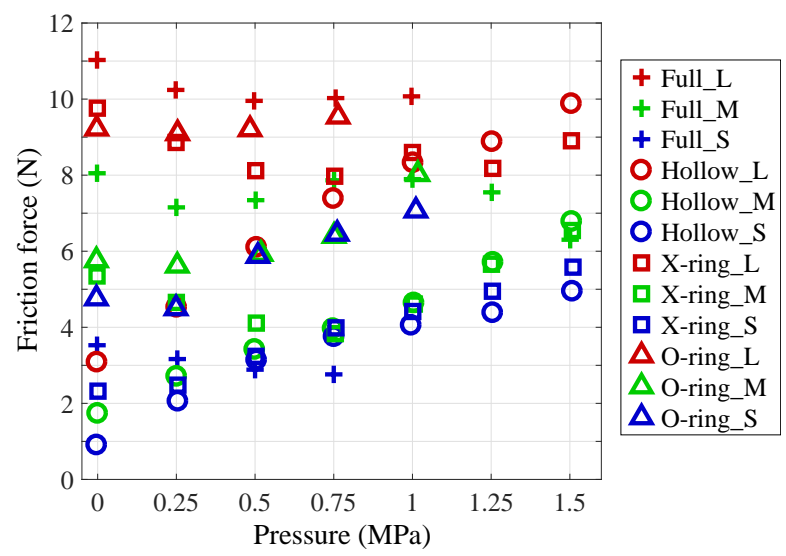

Fig. 11: Friction force for two pistons with different sealing geometries and sizes.

TABLE II: Identified mean values and standard deviations of force/pressure ratios for the different seal geometries

\begin{tabular}{|c||c|c|c|}
\hline Slope (N/MPa) & S & M & L \\
\hline Full & $12.4(0.6)$ & $10.6(0.9)$ & $8.8(0.3)$ \\
\hline Hollow & $12.4(0.1)$ & $11.8(0.2)$ & $10.9(0.4)$ \\
\hline X-ring & $11.1(0.3)$ & $9.6(0.5)$ & $8.3(0.6)$ \\
\hline O-ring & $12.7(0.1)$ & $11.3(0.4)$ & $11.3(0.7)$ \\
\hline
\end{tabular}

The theoretical value is equal to $13.2 \mathrm{~N} / \mathrm{MPa}$ if we consider the pressure as applied to a cylinder with diameter $4.1 \mathrm{~mm}$, which is the actual diameter of the polymer bearings after measurement.

Figure 11 shows the friction forces as evaluated during the dynamic assessment for all piston samples at velocity $1 \mathrm{~mm} / \mathrm{s}$. Two pistons are connected altogether during the experiment. The measured friction force is therefore twice the value for one piston. The influence of seal size is that the dynamic friction force increases with the seal diameter, as expected. The friction force values range from 0.5 to $11.0 \mathrm{~N}$, with a dependency of the pressure for most geometries. The lowest friction was measured for the Hollow $_{S}$ geometry, with friction ranging from $0.5 \mathrm{~N}$ at $0 \mathrm{MPa}$ to $4.9 \mathrm{~N}$ at 1.5 MPa. This means the friction per piston at pressure 1.5 $\mathrm{MPa}$ is $2.5 \mathrm{~N}$, while the theoretical force induced by pressure is $18.4 \mathrm{~N}$, which leaves an expected actuator force of $16 \mathrm{~N}, 87 \%$ of the theoretical maximum value. In addition, friction force with hollow pistons tends to increase with the pressure. This means we have, as expected during the design, an increase of lateral forces in the sealing when pressure is increased. For the Hollow $_{S}$ seal, the friction force is as low as $0.5 \mathrm{~N}$ at $0 \mathrm{MPa}$, which is of interest for fine no-load motions during positioning with a device using such hydraulic miniature pistons. We therefore selected this geometry for integration into the active revolute joint.

\section{INTEGRATION}

\section{A. Actuated joint integration}

The CAD of the actuated joint after integration of pistons is shown Figure 12. Two pistons are added to the gear rack

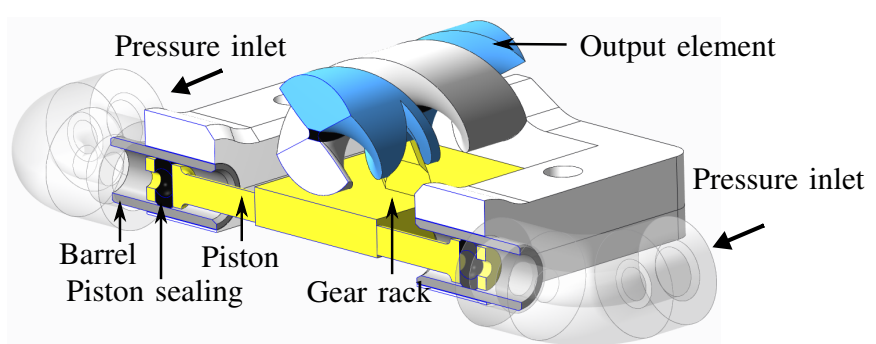

Fig. 12: CAD view of the active revolute joint with section to show the piston integration.

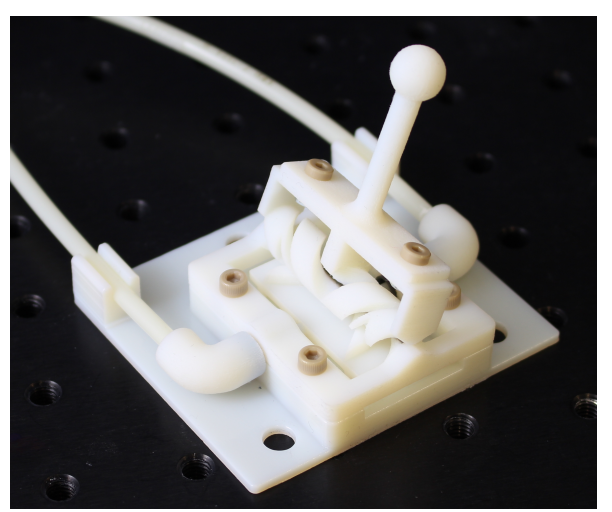

Fig. 13: Prototype of 3D-printed hydraulic revolute joint.

and the cylinder barrels are fixed to the base. A photograph of the active joint can be seen in Figure 13. Using the component, it is then straightforward to implement a compact and passive hydraulic master-slave system, as introduced in the attached video. The performance of the system is dependent on line pressurization, which has to be improved yet.

\section{B. Needle manipulation device}

As a final step, an illustration is implemented (Figure 1) to show the interest of the solution in terms of compactness and integration. We focus on a needle positioning device for MR-based needle insertions performed in interventional radiology. A 2-DOF needle manipulation system with remote center of motion is being implemented, following the parallel architecture preliminarily introduced in [20]. The two active joints are integrated on the base. The outer diameter of the device is less than $200 \mathrm{~mm}$, with a height of $70 \mathrm{~mm}$ and a weight of $225 \mathrm{~g}$. Thanks to MMAM, the system can be produced in only three main parts and constitutes a promising basis for developing a passive teleoperation platform (see attached video).

\section{Conclusion and Perspectives}

In this paper, a hydraulically-actuated compliant revolute joint is presented. MMAM is being used to design a compliant joint actuated by means of a rack-and-pinion system. The latter is controlled using hydraulic cylinders, for which new seal geometries have been considered and assessed. To the best of the authors knowledge, the designed elements, with 
4-mm pistons and 10-mm diameter joints constitute state-ofthe art solutions, that can in addition be used in challenging environment such as the MRI for medical applications. All the elements have been integrated to show the interest of the solutions with a needle manipulation prototype, passively controlled with a master interface.

Future work will be focused on pressure management in hydraulic lines and dynamic evaluation. Single-acting cylinders will be integrated to the systems for comparison. Finally, the proposed device will be further assessed in medical imagers given its relevance in interventional radiology.

\section{REFERENCES}

[1] C. R. Weiss, S. G. Nour, and J. S. Lewin. MR-guided biopsy: A review of current techniques and applications. Journal of Magnetic Resonance Imaging, 27(2):311-325, February 2008.

[2] A. Pfeil, L. Barbé, B. Wach, R. L. Cazzato, A. Gangi, and P. Renaud. Observations and experiments for the definition of a new robotic device dedicated to CT, CBCT and MRI-guided percutaneous procedures. In IEEE 41st International Engineering in Medicine and Biology Conference, 2018.

[3] D. Stoianovici, C. Jun, S. Lim, P. Li, D. Petrisor, S. Fricke, K. Sharma, and K. Cleary. Multi-Imager Compatible, MR Safe, Remote Center of Motion Needle-Guide Robot. IEEE Transactions on Biomedical Engineering, 65(1):165-177, 2017.

[4] J. P. Whitney, M. F. Glisson, E. L. Brockmeyer, and J. K. Hodgins. A low-friction passive fluid transmission and fluid-tendon soft actuator. In IEEE/RSJ International Conference on Intelligent Robots and Systems, pages 2801-2808, September 2014.

[5] D. Stoianovici, A. Patriciu, D. Petrisor, D. Mazilu, and L. Kavoussi. A New Type of Motor: Pneumatic Step Motor. IEEE/ASME Transactions on Mechatronics, 12(1):98-106, February 2007.

[6] Y. Chen, C. D. Mershon, and Z. T. H. Tse. A 10-mm MR-Conditional Unidirectional Pneumatic Stepper Motor. IEEE/ASME Transactions on Mechatronics, 20(2):782-788, April 2015.

[7] A. Pourghodrat and C. A. Nelson. Disposable Fluidic Actuators for Miniature In-Vivo Surgical Robotics. Journal of Medical Devices, 11(1):0110031-0110038, March 2017.

[8] J. Fras, Y. Noh, H. Wurdemann, and K. Althoefer. Soft fluidic rotary actuator with improved actuation properties. pages 5610-5615. IEEE, September 2017.

[9] J. P. Whitney, T. Chen, J. Mars, and J. K. Hodgins. A hybrid hydrostatic transmission and human-safe haptic telepresence robot. In IEEE International Conference on Robotics and Automation (ICRA), pages 690-695, May 2016.

[10] S. Xiaoming, S. Guangju, and L. Xin. Reconfigurable HydraulicMechanical Combined Drive Devices Based on Multi-functional Piston-Rod Built-in Hydraulic Cylinder. In International Conference on Digital Manufacturing Automation, volume 2, pages 134-137, December 2010.

[11] N. Burkhard, S. Frishman, A. Gruebele, J. P. Whitney, R. Goldman, B. Daniel, and M. Cutkosky. A rolling-diaphragm hydrostatic transmission for remote MR-guided needle insertion. In IEEE International Conference on Robotics and Automation (ICRA), pages 1148-1153, May 2017.

[12] S. Hashemi and W. K. Durfee. Low Friction, Long-Stroke Rolling Diaphragm Cylinder for Passive Hydraulic Rehabilitation Robots. In ASME Design of Medical Devices Conference, page V001T05A016, April 2017.

[13] C. Kundera and J. Bochnia. Investigating the stress relaxation of photopolymer O-ring seal models. Rapd Prototyping Journal, 20(6):533-540, 2014.

[14] N. Hungr, I. Bricault, P. Cinquin, and C. Fouard. Design and Validation of a CT- and MRI-Guided Robot for Percutaneous Needle Procedures. IEEE Transactions on Robotics, 32(4):973-987, August 2016.

[15] Z. Guo, Z. Dong, K. Lee, C. L. Cheung, H. Fu, J. D. L. Ho, H. He, W. Poon, D. T. Chan, and K. Kwok. Compact Design of a Hydraulic Driving Robot for Intraoperative MRI-Guided Bilateral Stereotactic Neurosurgery. IEEE Robotics and Automation Letters, 3(3):25152522 , July 2018.
[16] F. S. Farimani and S. Misra. Introducing PneuAct: ParametricallyDesigned MRI-Compatible Pneumatic Stepper Actuator. In IEEE International Conference on Robotics and Automation, pages 200205, 2018.

[17] A. Bruyas, F. Geiskopf, and P. Renaud. Design and Modeling of a Large Amplitude Compliant Revolute Joint: The Helical Shape Compliant Joint. Journal of Mechanical Design, 137(8):085003085003-8, August 2015.

[18] A. Pfeil, L. Barbé, B. Wach, A. Bruyas, F. Geiskopf, M. Nierenberger, and P. Renaud. A 3D-printed needle driver based on auxetic structure and inchworm kinematics. In ASME 2018 International Design Engineering Technical Conferences, 2018.

[19] S. P. Radzevich. Dudley's Handbook of Practical Gear Design and Manufacture, Second Edition. CRC Press, April 2012.

[20] A. Bruyas, F. Geiskopf, L. Meylheuc, and P. Renaud. Combining Multi-Material Rapid Prototyping and Pseudo-Rigid Body Modeling for a new compliant mechanism. In IEEE International Conference on Robotics and Automation (ICRA), pages 3390-3396, May 2014. 